\title{
ORIGINAL ARTICLE \\ Quantitative assessment of spinal cord perfusion by using contrast-enhanced ultrasound in a porcine model with acute spinal cord contusion
}

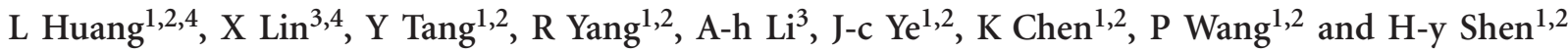

Objectives: To quantify spinal cord perfusion by using contrast-enhanced ultrasound (CEUS) in a porcine model with acute spinal cord injury.

Methods: Microcirculatory changes of acute spinal cord injury were shown by CEUS in a porcine model with spinal cord contusion at three selected time points, coupling with conventional ultrasound (US) and Color Doppler US (CDFI). Time-intensity curves and perfusion parameters were also obtained by autotracking contrast quantification (ACQ) software in the epicenter of contusion site, adjacent region and distant region, respectively. Neurologic and histologic examinations were used to confirm the severity of injury. Results: Conventional US revealed the spinal cord was hypoechoic and homogeneous, whereas the dura mater, pia mater and cerebral aqueduct were hyperechoic. On CDFI intramedullary blood vessels were displayed as segmental and columnar. It was homogeneous on CEUS. After spinal cord contusion, the injured region on gray scale US was hyperechoic. CDFI demonstrated intramedullary blood vessels of adjacent region had increased and dilated during the observation period. On CEUS the epicenter of contusion site was hypoperfusion, whereas its adjacent region was hyperperfusion compared with the distant region. Quantitative analysis showed that peak intensity decreased in epicenters of contusion but increased in adjacent regions significantly at all time points $(P<0.05)$. Evaluation of neurological function for post-contusion demonstrated significantly deterioration in comparison before injury $(P<0.05)$.

Conclusions: CEUS is a practical technique that provides overall views for evaluating microcirculatory pattern in spinal cord injury. Quantitative analysis shows the efficacy in assessment of perfusion changes after spinal cord injury.

Spinal Cord (2013) 51, 196-201; doi:10.1038/sc.2012.111; published online 9 October 2012

Keywords: spinal cord injury; perfusion; animal model; porcine; contrast-enhanced ultrasound

\section{INTRODUCTION}

A better understanding of spinal cord microcirculation is essential to further research in spinal cord injury. Although some methods, such as washout of radioactive indicators, ${ }^{1}$ implanted hydrogen electrode, ${ }^{2}$ the trapping of labeled microspheres, ${ }^{3}$ laser-Doppler flowmetry, ${ }^{2,4}$ closed spinal window ${ }^{5}$ and magnetic resonance imaging (MRI), ${ }^{6}$ have made significant contributions to the study of spinal cord blood flow (SCBF), these techniques have some shortcomings. The radioactive microsphere trapping method requires the killing of an animal and harvesting of tissue for the radioactivity counting. Thus, it cannot provide the real time information, and it usually needs many animals to get adequate data for analysis. A tiny delay in tissue harvesting will lead to a significant error for the mandatory timing. Although the laserDoppler technique is easy to use, non-invasive and recorded on-line in the assessment of microcirculatory changes of the brain and spinal cord, the major disadvantages are that the blood flow recorded is limited in a small tissue volume, the probe position on detecting point is hardly to consistently inter animals and the method is very sensitive to artifacts. ${ }^{7}$ In addition, the main drawback of closed spinal window with light microscopy or even with image-intensified highspeed video system (1000 frames $^{-1}$ ) is the depth of tissue penetration and so, this approach is restricted to observe the microcirculation in the spinal pia mater but not in the parenchyma of spinal cord. ${ }^{5,7}$ A typical non-invasive approach of MRI to measure perfusion is arterial spin labeling (ASL). The basic principle of ASL is that blood is magnetically labeled and serves as an intrinsic contrast agent. Because no contrast agent is needed, ASL techniques are completely non-invasive. For SCBF quantification ASL as the alternative method for absolute quantitative perfusion measurement might suffer from too low sensitivity. Recently, Weber et al. ${ }^{8}$ have presented a first report of quantitative SCBF measurements in mice by MRI with a pulsed ASL technique based on a presaturated flowsensitive alternating inversion recovery (presat-FAIR) EPI sequence. However, MRI is not a continuous real-time perfusion.

US has recently benefited from the introduction of contrast agents. ${ }^{9}$ These agents consist of microspheres that enhance ultrasonic reflection signals and modify acoustic properties of regions of

${ }^{1}$ Department of Orthopedics, Sun Yat-sen Memorial Hospital, Sun Yat-sen University, Guangzhou, China; ${ }^{2}$ Institute of Spinal Cord Injury, Sun Yat-sen University, Guangzhou, China and ${ }^{3}$ Department of Ultrasound, Cancer Center of Sun Yat-sen University, State Key Laboratory of Oncology in Southern China, Guangzhou, China

Correspondence: Dr L Huang, or Dr H-y Shen, Department of Orthopedics, Sun Yat-sen Memorial Hospital, Sun Yat-sen University or Institute of Spinal Cord Injury, Sun Yat-sen University, Guangzhou, China.

E-mail: shenhuiyong@yahoo.com.cn or hakkahuanglin@gmail.com.cn

${ }^{4}$ These authors contributed equally to this work.

Received 24 October 2011; revised 15 July 2012; accepted 18 August 2012; published online 9 October 2012 
interest. The second generation echo-contrast agent SonoVue (Bracco, Milan, Italy) consists of microbubbles stabilized by phospholipids and filled with sulphur hexafluoride (SF6). It has a mean diameter of $2.5 \mu \mathrm{m}$ and is a blood pool imaging agent. Therefore, contrast-enhanced ultrasound (CEUS) has made microvascular imaging possible. ${ }^{10,11}$ In comparison with those methods mentioned above, CEUS have a lot of advantages, including low costs, better availability, subject (patient) comfort, and no exclusion of patients or animals with certain indwelling metals (internal fixation) and bedside measurement, for example, for intraoperative animals or patients. What's more, CEUS enables the simultaneous measurement of tissue perfusion (microcirculation) in real time.

Scientists have long used animal spinal cord contusion models to study the pathophysiology of spinal cord injury. ${ }^{12}$ Contusion models had an important role in the discovery of progressive secondary tissue damage and most therapies that have gone to human clinical trial were first validated in spinal cord contusion models. There is a linear relationship between force and displacement and that both of these factors have a role in determining the anatomical and functional deficits after spinal cord contusion injury. ${ }^{13}$

The purposes of this study were to evaluate the role of CEUS in microcirculation of acute spinal cord contusion, and to investigate its perfusion characteristics of CEUS.

\section{MATERIALS AND METHODS}

\section{Study design}

Six juvenile, domestic female pigs, 3 months of age and weighing $15-16 \mathrm{~kg}$, were subjected for this experiment. Spinal cord contusions of the animals were produced by a modified spinal cord impactor system. CEUS was performed to monitor microcirculatory changes pre and post spinal cord contusions. All procedures were approved by the Ethics Committee and the Animal Experimentation Committee of Sun Yat-sen Memorial Hospital, Sun Yat-sen University, and conducted in accordance with the Public Health Service Guide for the Care and Use of Laboratory Animals.

\section{Anesthesia and perioperative management}

After pretreatment with $15 \mathrm{mg} \mathrm{kg}^{-1} \mathrm{ketamine}$ and $0.03 \mathrm{mg} \mathrm{kg}^{-1}$ atropine given intramuscularly to induce the anesthesia, animals were further anesthetized with intravenous Thiamylal $\left(1.5 \mathrm{mg} \mathrm{kg}^{-1}\right)$ administered slowly. Intramuscular buprenorphine $\left(0.1 \mathrm{mg} \mathrm{kg}^{-1}\right)$ was used as an analgesic. The antibiotic Ceftiofur $\left(3 \mathrm{mg} \mathrm{kg}^{-1}\right)$ was given intramuscularly to prevent bacterial infection.

\section{Porcine model of spinal cord contusion}

After a deep level of anesthesia, the pig was placed prone on the table and a midline dorsal incision was made in the skin from $\mathrm{T}_{8}$ level to $\mathrm{T}_{13}$. The muscles around $\mathrm{T}_{8}$ to $\mathrm{T}_{13}$ were incised and retracted on both sides of the vertebral column to expose spinous processes and laminas. Total laminectomy of $T_{9}$ to $T_{12}$ was performed with preservation of the dura. Spinous processes of $T_{8}$ and $\mathrm{T}_{13}$ were reserved for clamping with the modified spinal cord impactor system. The thoracic spinal cord contusion model was induced with a modified weight-drop impactor $(50 \mathrm{~g}$ weight, dropped from a height of $15 \mathrm{~cm}$ ).

\section{US imaging protocol, contrast agent and injection technique}

First of all, before contusion, conventional gray-scale US and Color Doppler US (CDFI) were performed on the exposed thoracic cord. Real-time CEUS using low mechanical index (0.25) started as soon as the bolus injection of contrast agent was performed. An intravenous bolus injection of $2.0 \mathrm{ml}$ of SonoVue was used, followed by a flush of $10 \mathrm{ml}$ of saline. Images and clips lasting for $70 \mathrm{~s}$ were stored on hard disk for online analysis.

Then, the thoracic spinal cord of the animal was injured by a modified spinal impactor system $(50 \mathrm{~g}$ weight, dropped from a height of $15 \mathrm{~cm}$ ). The impactor was immediately removed from the injured spinal cord. The US examination procedures were performed again. Three time points were selected to demonstrate the microperfusion after spinal cord contusion: $0 \mathrm{~min}$ (instantly), $30 \mathrm{~min}$ and $120 \mathrm{~min}$.

CEUS was performed using Acuson Sequoia 512 (Siemens, Mountain View, CA, USA), equipped with $15 \mathrm{~L} 8 \mathrm{w}-\mathrm{S}$ transducer, frequency $7.0 \sim 10.0 \mathrm{MHz}$, and contrast pulse sequencing software: ACQ. All US examinations were performed by one radiologist (Xi Lin, with 5 years of experience in CEUS).

\section{Quantitative analysis in CEUS}

ACQ software was applied to derive the clips. Three regions of interest were set in epicenters of contusion site in injured thoracic cord, adjacent regions and distant regions, which were $\sim 1.5 \mathrm{~cm}$ far from the injured site. Time-intensity curves and perfusion parameters: peak intensity (PI) were obtained by ACQ software from regions of interest. Images obtained with the sequences were analyzed by one radiologist (An-hua Li, with 5 years of experience in CEUS).

\section{Postoperative management}

After incision was closed, the pig was placed in a separate pen as soon as it was alert. Food and water were provided starting on the first postoperative day (POD). Analgesic treatment (buprenorphine $0.1 \mathrm{mg} \mathrm{kg}^{-1}$ ) was maintained for all 7 PODs.

\section{Neurological evaluation}

All animals survived the operative procedure and completed the full observation period of 7 PODs. To confirm the severity of the neurological deficit after spinal cord contusion, neurological examination, using the Tarlov score, ${ }^{14}$ was employed on POD 2 and POD 7 simultaneously by two investigators. The modified Tarlov Scoring was a four-point scale that depicts no locomotion as 0 points and a normal gait as 4 points. After assessment of the Tarlov score on POD 7, the animals were killed. Histological observation was performed to show the severity of injury to the spinal cord. The hematoxylin and eosin stained sections were transfer to digital images under microscopy. Then the percentage of lesion tissue area compared with the whole cord cross-sectional area was measured by using Adobe Photoshop CS3 (Adobe, San Jose, CA, USA).

\section{Data analysis}

All values obtained were expressed as the mean \pm s.d. One-way ANOVA test was performed in comparison of PI after contusion by $\mathrm{G}^{*}$ power software (Düsseldorf, Germany). A $P<0.05$ was considered to be statistically significant.

\section{RESULTS}

\section{CEUS and ACQ analysis}

Precontusion. Conventional US revealed the subdural space and the spinal cord in sagittal plane to be hypoechoic and homogeneous, whereas the dura mater, pia mater and cerebral aqueduct were hyperechoic (Figure 1a). On CDFI in sagittal plane intramedullary blood vessels were divided into dorsal and ventral parts with reverse blood flow direction by cerebral aqueduct. They were displayed as segmental and columnar (Figure 1b). After contrast agent administration, the enhanced characteristic of the spinal cord was homogeneous (Figure 4a).

Postcontusion. Conventional gray scale US demonstrated that the injured region was hyperechoic. There was a corresponding grow in the area of hyperechoicity during the observation period (Figures $2 \mathrm{a}$ and b). The injured spinal cord was swelling. The architecture distortions of the dura mater, pia mater and cerebral aqueduct were also displayed. CDFI demonstrated intramedullary blood vessels of adjacent region had increased and dilated during the observation period (Figures $2 \mathrm{c}$ and 3 ).

On CEUS the enhanced characteristic of contused spinal cord was heterogeneous (Figures $2 \mathrm{~d}$ and $4 \mathrm{~b}-\mathrm{d}$ ). The epicenter of contusion site which was hyperechoic region on gray scale US was hypoperfusion, while its adjacent region was hyperperfusion and the distant spinal 
cord was moderately enhanced on CEUS (Figure 4). In comparison of distant regions, quantitive analysis showed that PI decreased in epicenters of contusion but increased in adjacent regions significantly at all time points $(P<0.05)$ (Figure 5$)$

\section{Functional evaluation}

Figure 6 displayed the neurological function recovery of the animals. None of the six animals recovered from the protocol with normal neurological function, with a Tarlov score of 4 on the morning after surgery. All animals emerged a Tarlov score from 0-2 on POD 2, and remained a same range on POD 7,which was significantly deteriorated in comparison of normal function $(P<0.05)$. Compared with the

\section{a}

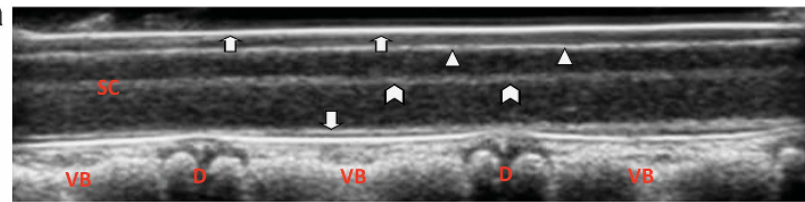

b

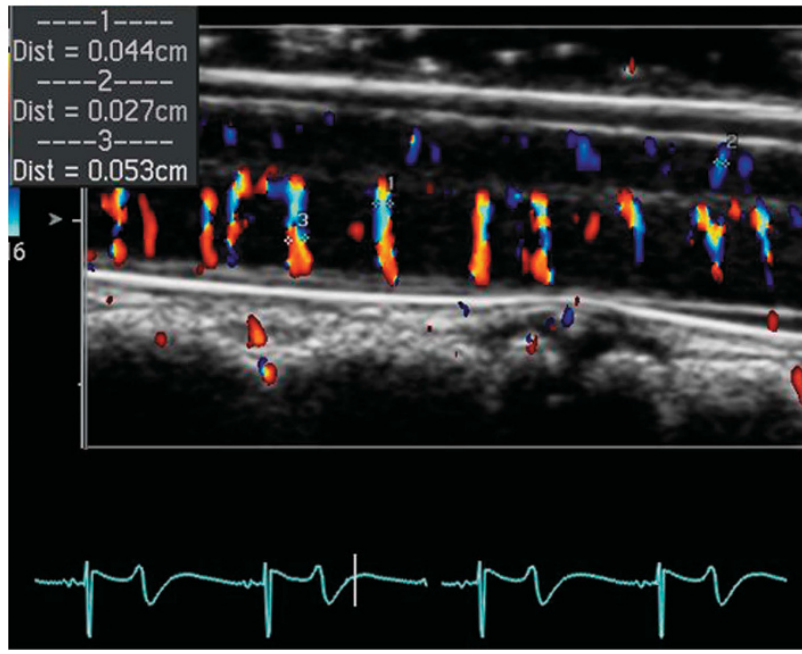

Figure 1 Sonography of uninjured spinal cord (longitudinal view): (a) Gray scale and (b) CDFI of uninjured spinal cord. SC, spinal cord; VB, vertebrae body; $\mathrm{D}$, disc. Ч $\_$indicate dura mater of spinal cord; $\Delta$ indicates spinal pia mater; $\boldsymbol{\omega}$ indicates cerebral aqueduct.
POD 2, no significant recovery was observed during the 7 days after contusion $(P>0.05)$.

\section{Histological examination}

Histology imaging confirmed the degree of contusion (Figure 7). Serious damage such as hemorrhaging or cavity formation was observed on hematoxylin and eosin stain sections.

\section{DISCUSSION}

In this study, CEUS provided advantages in assessment on microcirculatory changes within spinal cord in a pig model. Hyperfusion was shown in adjacent region and hypofusion in the epicenter of contusion on CEUS, as compared with distant region. The objective observation was confirmed by ACQ analysis. Previously, SCBF was evaluated by hydrogen clearance method, radioactive tracer microsphere technique or laser-Doppler flowmetry technique in literatures. Laser Doppler was a popular real-time technique for SCBF. However, it could only measure SCBF within superficial tissues in large animals because of its limited detecting ability, which was only up to a 3-mm depth of spinal cord. ${ }^{15}$ The method was also too sensitive in artifacts. ${ }^{4}$ Moreover, it could not provide an overall view of spinal cord microperfusion. In contrast, this study suggested that CEUS was easy to be performed. It provided advantages of real-time microperfusion, an overall view and non-radiation. Therefore, it was useful for evaluating microperfusion of spinal cord.

To date, a comparison between CEUS and perfusion MRI in spinal cord is lacking. A typical non-invasive MRI approach to measure perfusion is ASL. The basic principle of ASL is that blood is magnetically labeled and serves as an intrinsic contrast agent. Because no contrast agent is needed, ASL techniques are completely noninvasive. For SCBF quantification, ASL as the alternative method for absolute quantitative perfusion measurement might suffer from too low sensitivity. Recently, Duhamel et al. ${ }^{6}$ have presented a first report of quantitative SCBF measurements in mice by MRI with a pulsed ASL technique based on a presaturated flow-sensitive alternating inversion recovery (presat-FAIR) EPI sequence. In comparison with those methods, such as laser-Doppler flowmetry or MRI, CEUS have a lot of advantages, including low costs, better availability, subject (patient) comfort, and no exclusion of patients or animals with certain indwelling metals (internal fixation) and bedside measurement, for example, for intraoperative animals or patients. What's more, compared with MRI, CEUS enable the simultaneous measurement of tissue perfusion in real time.
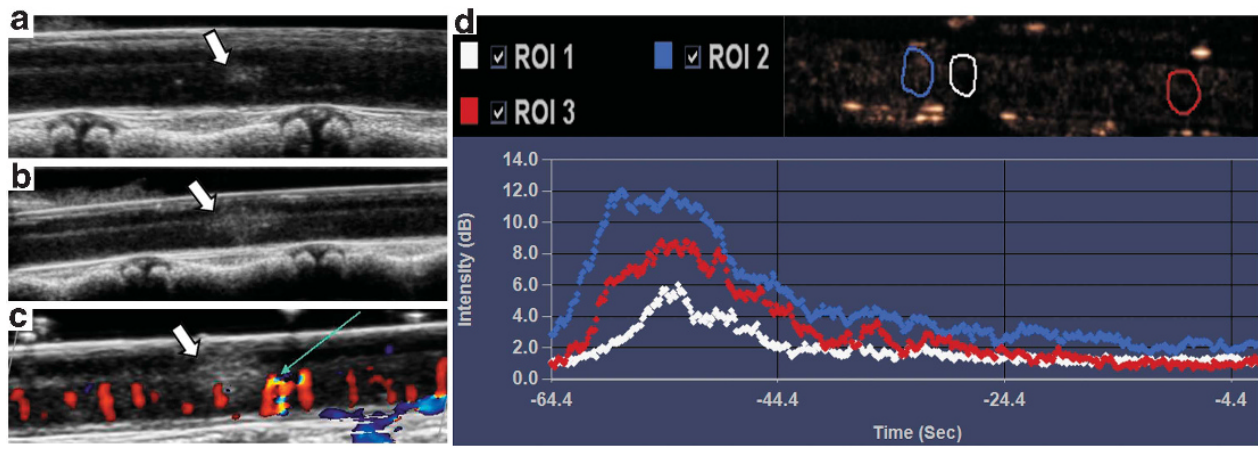

Figure 2 (a, b) Gray-scale sonography of spinal cord immediately post injury demonstrates increases in lesion area in 1 min. (c) CDFI demonstrated the dilated intramedullary blood vessels of injured region (arrow). (d) Representative CEUS and ACQ analysis showed the pattern of ischemic areas in contusion site after SonoVue infusion and enhancement in adjacent region. そ indicated contusive lesion, long arrow indicated dilated vessels in adjacent region. ROI (regions of interest) $1, \mathrm{ROI} 2$, and ROI 3 represented the epicenter of contusion site, adjacent region and distant region, respectively. 

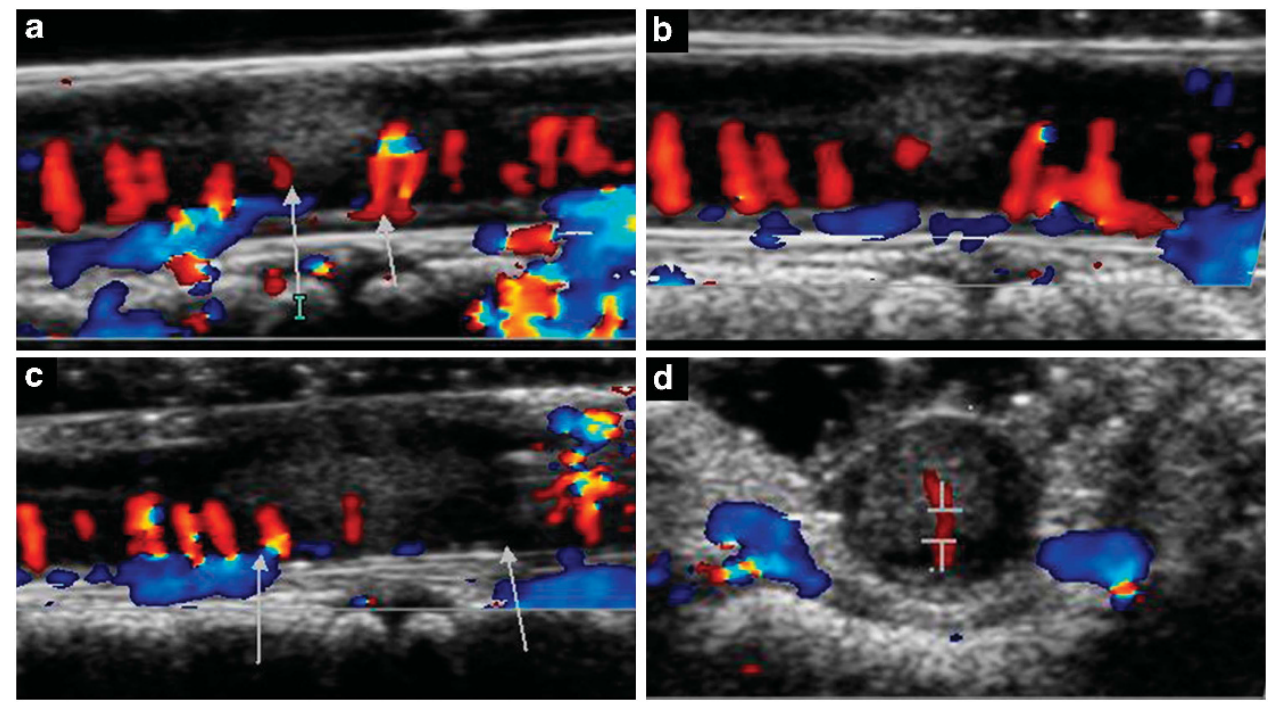

Figure $3 \mathrm{CDFI}$ of contusived spinal cord during time course. (a) 0 min (instantly after contusion); (b) 30 min after contusion; (c) longitudinal view of the contusive cord at $120 \mathrm{~min}$ after injury and (d) axial view of the contusive cord at $120 \mathrm{~min}$ after injury.
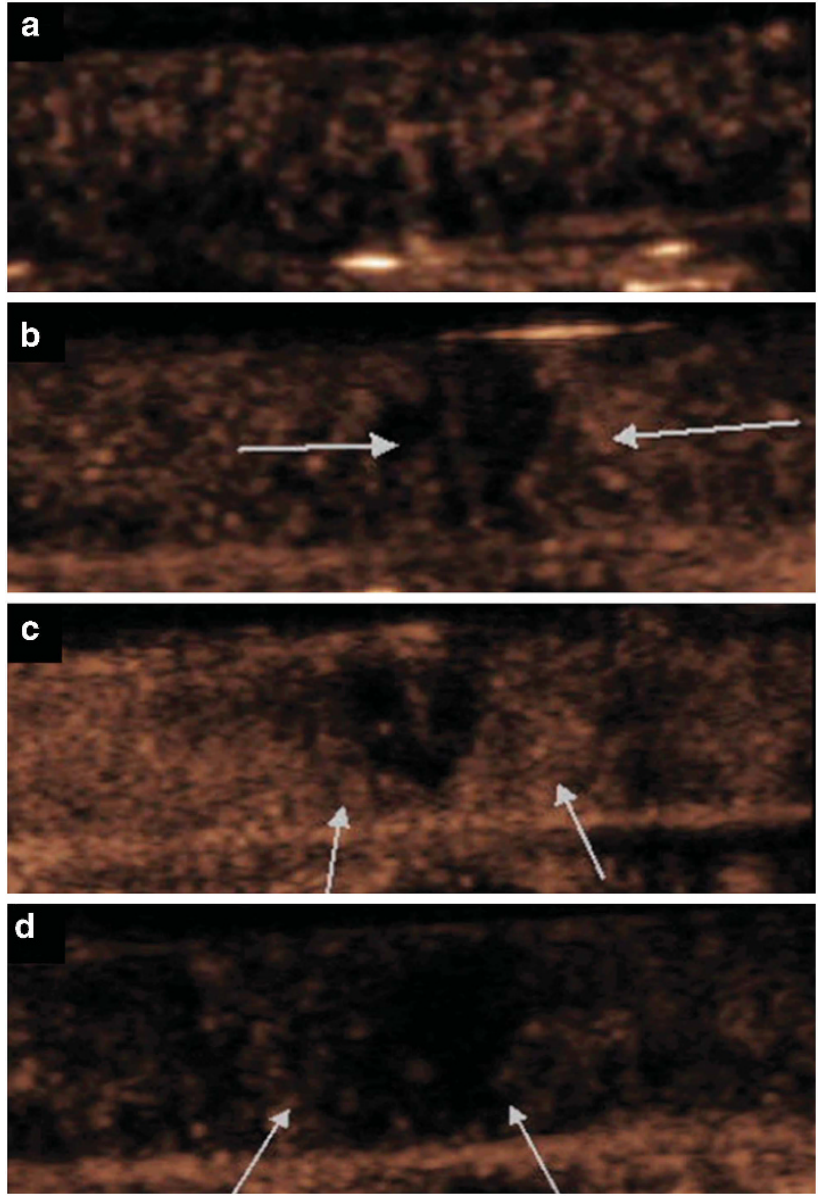

Figure 4 Representative CEUS pictures of the spinal cord: (a) uninjured spinal cord; (b) 0 min after contusion; (c) $30 \mathrm{~min}$ after contusion; (d) 120 min after contusion.

On gray scale US the epicenter was hyperechoic. There was a corresponding grow in the area of hyperechoicity during the experimental session. The latter finding suggested secondary injury. Less

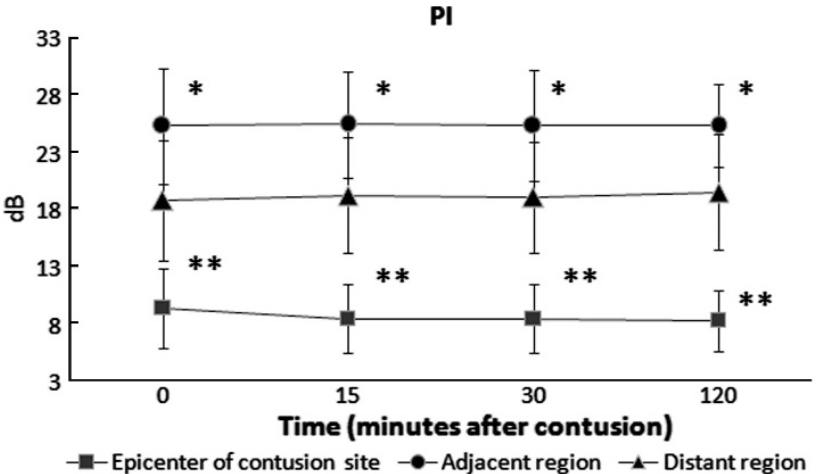

Figure 5 Diagram showed the PI was markedly decreased in the epicenter of contusion but increased significantly in the adjacent region, as compared with distant region. ${ }^{*} P<0.05,{ }^{* *} P<0.01$.

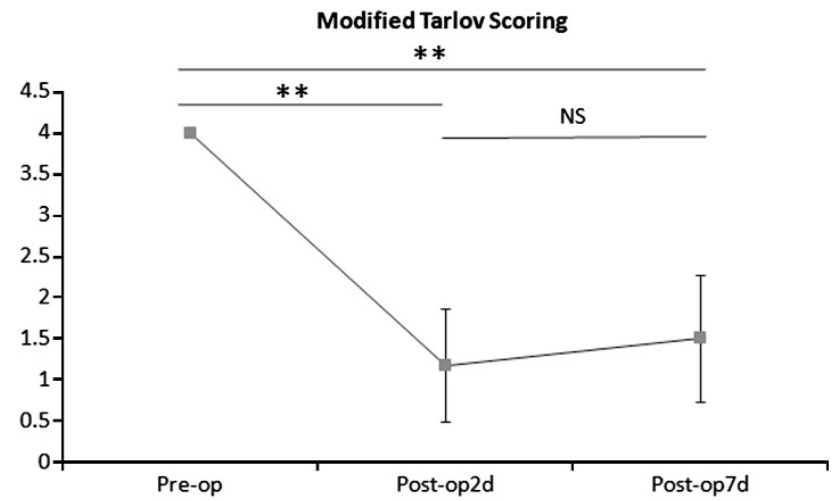

Figure 6 Neurological function of the animals evaluated by modified Tarlov scoring scale. Deterioration in neurological function was significantly emerged and no obviously recovery was observed during 7 days after contusion. ${ }^{*} P<0.05,{ }^{*} P<0.01$; NS: no statistical significant. A full color version of this figure is available at the Spinal Cord journal online.

blood flow was observed in epicenter on CDFI. Using CEUS, its enhancement pattern was hyperechoic and quantitative analysis showed that PI of the epicenter was significantly lower than adjacent 

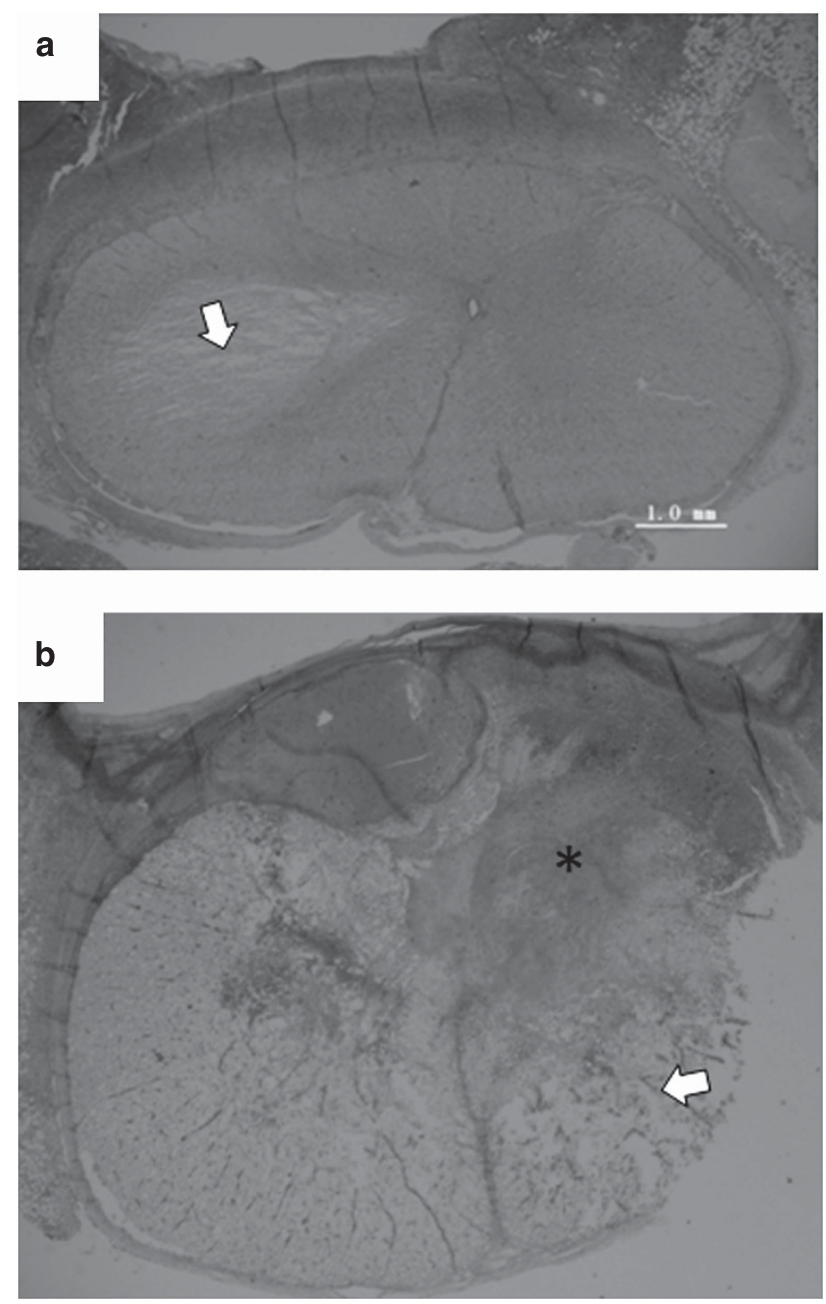

C

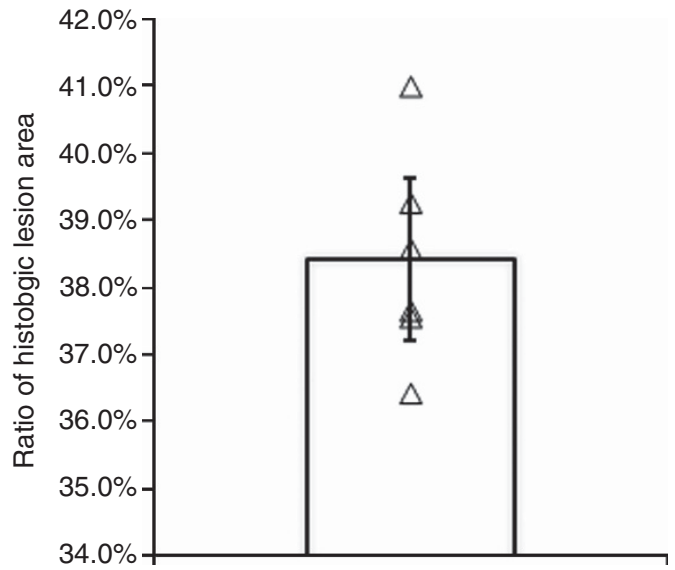

Figure 7 (a, b) Photomicrograph of representative hematoxylin and eosinstained spinal cord cross-section, showing degrees of tissue damage in response to the injury severity. Magnification bars are indicated. (c) Ratio of histological lesion area of spinal cord. $\square$ indicate necrosis and cyst formation or micro cysts of injured spinal cord; * indicate hemorrhage of injured spinal cord. A full color version of this figure is available at the Spinal Cord journal online.

and distant regions throughout this period $(P<0.05)$. This result in general was consistent with what was previously reported. ${ }^{16}$ The most significant difference from previous studies was the perfusion in adjacent regions. A majority of studies reported significant posttraumatic hypoperfusion in adjacent segments of spinal cord. ${ }^{17-19}$ Likewise, a number of clinical and experimental studies had shown less cerebral blood flow in and around contused brain parenchyma. ${ }^{20,21}$ However, this study showed that on CDFI and CEUS there was a correspondingly significant hypervascularity and hyperperfusion in the adjacent region, which at least lasted $120 \mathrm{~min}$. Quantitative analysis confirmed this observation. These findings provided more information on changes of perfusion in spinal cord injury.

Acute spinal cord injury resulted in the initial physical disruption of structures in the spinal cord and in the generation of secondary events that collectively injure intact and neighboring tissue. Microcirculatory events had a key role in both primary and secondary injury mechanisms. Early hypoperfusion showed obviously on CEUS and by quantitative analysis in contusion site. One possible explanation was that the initial impact disrupted the blood supplies within the spinal cord and resulted in local infarction caused by hypoxia and ischemia. ${ }^{22}$ Moreover, hypoperfusion frequently indicated vascular damage from immediate vasospasm of superficial vessels to intraparenchymal hemorrhage and the formation of thromboses. ${ }^{21,23}$ Hyperperfusion in pericontusion area were ascribed to an increasing volume of blood flow, dilation of microvessels, loss of autoregulation or opening of an arteriovenous shunt. In a moderate controlled cortical impact injury model, mean density and diameter of cortical microvessels were significantly reduced and increased, respectively, in the contusion margin at the initial time points. ${ }^{24}$ In our study, there was significant difference in vessels dilation on CDFI and hyperperfusion on CEUS in adjacent region. These vivid and realtime features by CDFI and CEUS provided strong evidence that vessel dilation in pericontusional region was a response to the mechanical insult and metabolic demand. The latter was due to acute increasing in oxygen consumption ${ }^{25}$ and glucose metabolism after trauma. ${ }^{26}$

There were several limitations in this study. First of all, because of the attenuation of bone, CEUS could not be performed in spinal cord canal as it interfered with the integrity of spinal lamina and it could not be used after closed incision. Special probe is needed for spinal cord in the future. Second, it was small sample and there were lack of graded models on spinal cord injury in this study.

Microbubble contrast agents are currently implemented in a variety of both clinical and preclinical US imaging studies. ${ }^{27}$ The therapeutic and diagnostic capabilities of these contrast agents are ongoing and show potential clinical prospects. ${ }^{28}$ Most human spinal cord injuries result from fracture and dislocation of the spinal column. Spinal cord contusion models have had a significant contribution to the field. ${ }^{12}$ Standardized procedure could help in confirmation of the severity of contusive spinal cord. The neurological results and the histological imaging from our study indicated that all pigs received moderatesevere contusion. Therefore, it is possible to evaluate the efficacy of this technique in clinical applications, including traumatic or iatrogenic spinal cord injury. What's more, it is deserved to investigate CEUS thoroughly in combination with neuroprotective or therapeutic genes or drugs for spinal cord injury in the future.

\section{CONCLUSIONS}

CEUS is a practical technique that provides overall views for evaluating microcirculatory pattern in spinal cord injury. Quantification analysis shows the efficacy in assessment of perfusion changes after spinal cord injury.

\section{DATA ARCHIVING}

There were no data to deposit. 


\section{CONFLICT OF INTEREST}

The authors declare no conflict of interest.

\section{ACKNOWLEDGEMENTS}

We thank the National Natural Science Foundation of China (grant no. 30901506, 30973033) and the Provincial Natural Science Foundation of Guangdong (grant no. 2010B060500021 and 8151008901000094).

1 Weir CJ, Zivin JA, Lyden PD. Inter-relationships between spinal cord blood flow, neuronal death and neurological function in rabbit spinal cord ischemia. Brain Res 2002; 946: 43-51.

2 Ueda Y, Kawahara N, Tomita K, Kobayashi T, Murakami H, Nambu K. Influence on spinal cord blood flow and function by interruption of bilateral segmental arteries at up to three levels: experimental study in dogs. Spine 2005; 30: 2239-2243.

3 Maeda M, Inoue M, Takao S, Ikegami Y, Nakai M, Krieger AJ et al. Chemical stimulation of the nucleus tractus solitarii decreases spinal cord blood flow in anesthetized rats. Neurosci Lett 1995; 185: 111-114.

4 Westergren $\mathrm{H}$, Farooque M, Olsson Y, Holtz A. Spinal cord blood flow changes following systemic hypothermia and spinal cord compression injury: an experimental study in the rat using Laser-Doppler flowmetry. Spinal Cord 2001; 39: 74-84.

5 Ishikawa M, Sekizuka E, Oshio C, Sato S, Yamaguchi N, Terao S et al. Platelet adhesion and arteriolar dilation in the photothrombosis: observation with the rat closed cranial and spinal windows. J Neurol Sci 2002; 194: 59-69.

6 Duhamel G, Callot V, Cozzone PJ, Kober F. Spinal cord blood flow measurement by arterial spin labeling. Magn Reson Med 2008; 59: 846-854.

7 Frerichs KU, Feuerstein GZ. Laser-Doppler flowmetry. A review of its application for measuring cerebral and spinal cord blood flow. Mol Chem Neuropathol 1990; 12: 55-70.

8 Weber M-A, Krix M, Delorme S. Quantitative evaluation of muscle perfusion with CEUS and with MR. Eur Radiol 2007; 17: 2663-2674.

9 Cosgrove D. Ultrasound contrast agents: an overview. Eur J Radiol 2006; 60: 324-330.

10 Weber MA, Krix M, Delorme S. Quantitative evaluation of muscle perfusion with CEUS and with MR. Eur Radiol 2007; 17: 2663-2674.

11 Bartels E, Henning S, Wellmer A, Giraldo-Velasquez M, Kermer P. Evaluation of cerebral perfusion deficit in stroke patients using new transcranial contrast imaging CPS technology-preliminary results. Ultraschall Med 2005; 26: 478-486.
12 Young W. Spinal cord contusion models. Prog Brain Res 2002; 137: 231-255.

13 Nader G, Bradley JK, David S. Tissue displacement and impact force are important contributors to outcome after spinal cord contusion injury. Exp Neurol 2005; 196: 9-17.

14 Tarlov IM. Acute spinal cord compression paralysis. J Neurosurg 1972; 36: 10-20.

15 Hamamoto Y, Ogata T, Morino T, Hino M, Yamamoto H. Real-time direct measurement of spinal cord blood flow at the site of compression: relationship between blood flow recovery and motor deficiency in spinal cord injury. Spine 2007; 32: 1955-1962.

16 Tozaki M, Isobe S, Yamaguchi M, Ogawa Y, Kohara M, Joo C et al. Optimal scanning technique to cover the whole breast using an automated breast volume scanner. Jpn J Radiol 2010; 28: 325-328.

17 Fehlings MG, Tator CH, Linden RD. The effect of nimodipine and dextran on axonal function and blood flow following experimental spinal cord injury. J Neurosurg 1989; 71: 403-416.

18 Guha A, Tator $\mathrm{CH}$, Rochon J. Spinal cord blood flow and systemic blood pressure after experimental spinal cord injury in rats. Stroke 1989; 20: 372-377.

19 Kapadia SE. Ultrastructural alterations in blood vessels of the white matter after experimental spinal cord trauma. J Neurosurg 1984; 61: 539-544.

20 Schroder ML, Muizelaar JP, Fatouros P, Kuta AJ, Choi SC. Early cerebral blood volume after severe traumatic brain injury in patients with early cerebral ischemia. Acta Neurochir Suppl 1998; 71: 127-130.

21 Tenjin H, Ueda S, Mizukawa N, Imahori Y, Hino A, Yamaki T et al. Positron emission tomographic studies on cerebral hemodynamics in patients with cerebral contusion. Neurosurgery 1990; 26: 971-979.

22 Dumont RJ, Okonkwo DO, Verma RS, Hurlbert RJ, Boulos PT, Ellegala DB et al. Acute spinal cord injury, part I: Pathophysiologic mechanisms. Clin Neuropharmacol 2001; 24: 254-264.

23 Tornheim PA, McDermott F, Shiguma M. Effect of experimental blunt head injury on acute regional cerebral blood flow and edema. Adv Neurol 1990; 52: 377-384.

24 Chen S, Pickard JD, Harris NG. Time course of cellular pathology after controlled cortical impact injury. Exp Neurol 2003; 182: 87-102.

25 Levasseur JE, Alessandri B, Reinert M, Bullock R, Kontos HA. Fluid percussion injury transiently increases then decreases brain oxygen consumption in the rat. $J$ Neurotrauma 2000; 17: 101-112.

26 Perthen JE, Lansing AE, Liau J, Liu TT, Buxton RB. Caffeine-indcued uncoupling of cerebral blood flow and oxygen metabolism: A calibrated BOLD fMRI study. Neuroimage 2008; 40: 237-247.

27 Liu Z, Lammers T, Ehling J, Fokong S, Bornemann J, Kiessling F et al. Iron oxide nanoparticle-containing microbubble composites as contrast agents for MR and ultrasound dual-modality imaging. Biomaterials 2011; 32: 6155-6163.

$28 \mathrm{Hu}$ YZ, Zhu JA, Jiang YG, Hu B. Ultrasound microbubble contrast agents: application to therapy for peripheral vascular disease. Adv Ther 2009; 26: 425-434. 